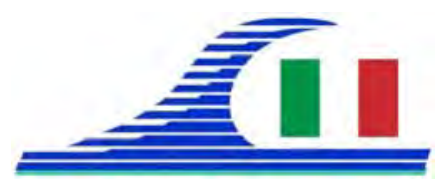

Conférence Méditerranéenne Côtière et Maritime EDITION 3, FERRARA, ITALIA (2015)

Coastal and Maritime Mediterranean Conference

Disponible en ligne - http://www.paralia.fr - Available online

\title{
Etude du fonctionnement hydro-sédimentaire de la zone portuaire de Sidi Bou Saïd, Tunisie
}

\author{
Wissem GHARBI ${ }^{1}$, Noomane HAOUEL ${ }^{1}$, Hedia SALHI ${ }^{1}$, \\ Pierre FARNOLE $^{2}$
}

\author{
1. MARITEC, Appnt BM3 Immeuble Luxor 2, 1007 Montplaisir Tunis, Tunisie. \\ maritec@planet.tn \\ 2. ERAMM, 1503 rte des Dolines, BP 42, 06901 Sophia Antipolis, France. \\ pf@eramm.fr
}

\section{Résumé :}

Le port de Sidi Bou Saïd connaît depuis sa création un problème d'ensablement aigu nécessitant le recours à des dragages périodiques de son accès et entravant le fonctionnement normal du port (COMETE ENGINEERING, 2008; STUDISOGREAH, 2003). L'état du port et de ses environnements justifie la réalisation d'une étude hydrosédimentaire pour comprendre le fonctionnement de la courantologie de la zone portuaire de Sidi Bou Saïd, analyser le phénomène d'ensablement de la passe d'entrée au port et vérifier l'impact des aménagements proposés. Une chaîne de calcul de modélisation hydrosédimentaire globale a été mise en place.

Mots-clés : Ouvrage, Aménagement côtier, Dynamique littorale, Modélisation numérique, Hydrodynamique, Transport sédimentaire.

\section{Introduction}

Situé à Cap Carthage, dans le Golfe de Tunis, à une vingtaine de kilomètres au NordEst de Tunis, le port de plaisance est adossé à la colline de Sidi Bou Saïd (voir figure1). La construction du port a débuté en 1962, l'achèvement de la digue principale Nord en 1965 et de la digue secondaire Sud en 1970.

Le port est formé par une digue principale à l'Est, d'une longueur de $400 \mathrm{~m}$, et une contre-digue à l'Ouest, d'une longueur d'environ $220 \mathrm{~m}$. Au sud, une plage s'est formée contre cette contre-digue.

L'accès au port est orienté Sud-Ouest et possède une ouverture de $60 \mathrm{~m}$. Le bassin portuaire est divisé en une zone Est et une zone Ouest par un môle central. La profondeur initiale lors de la construction était de -4.5 m NGT (Nivellement Général de la Tunisie).

Le port de Sidi Bou Saïd connaît depuis sa création un problème d'ensablement aigu (voir figure 2), estimé à $5000 \mathrm{~m}^{3} / \mathrm{an}$, nécessitant le recours à des dragages périodiques de son accès et entravant le fonctionnement normal du port. Une gêne liée à l'agitation est également relevée au niveau de la passe d'entrée et des ouvrages d’accostage. 
Côtes méditerranéennes menacées :

Risques et défis dans le contexte du changement climatique
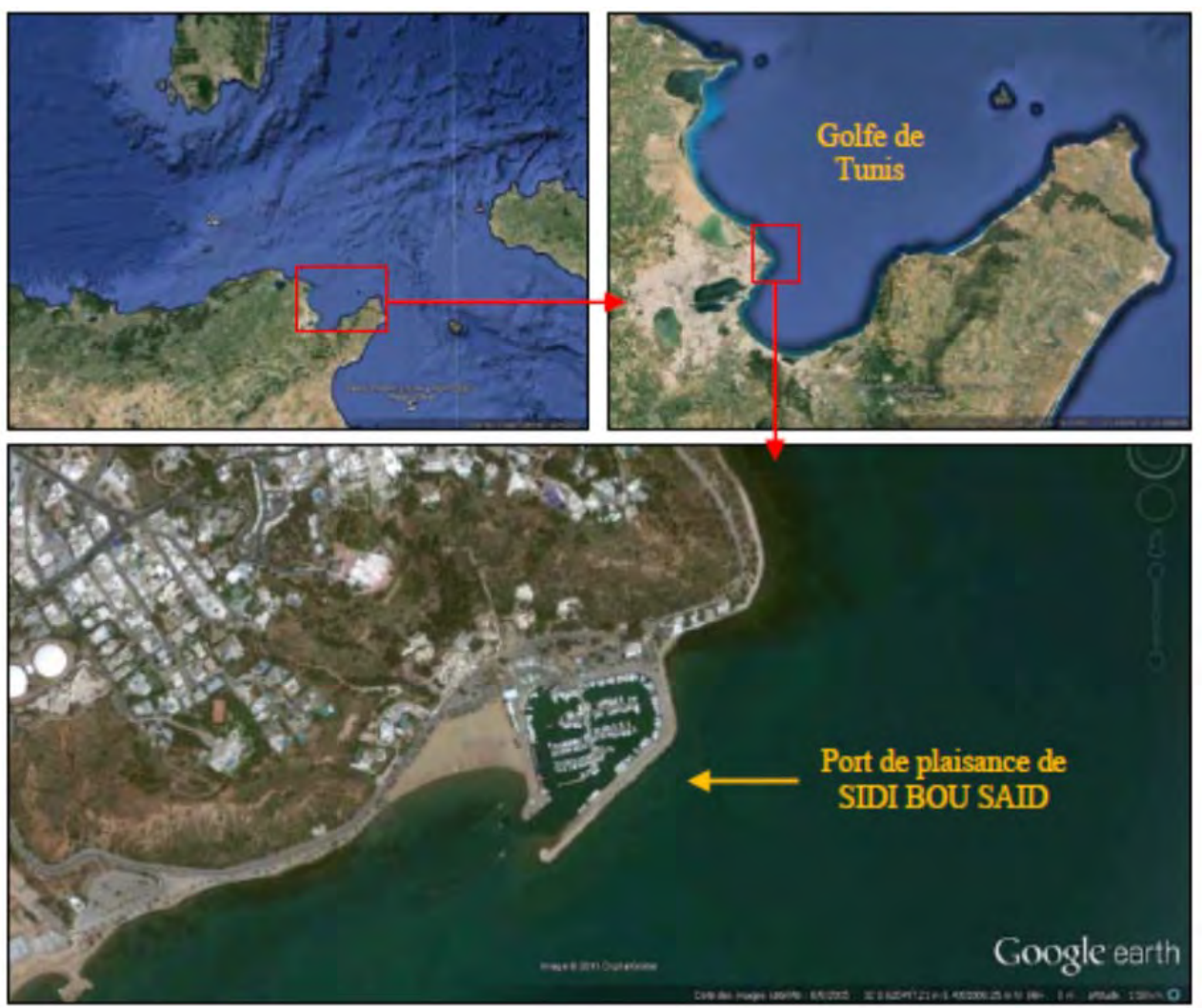

Figure 1. Localisation de la zone d'étude

(Images satellitaires, Google Earth 2015).

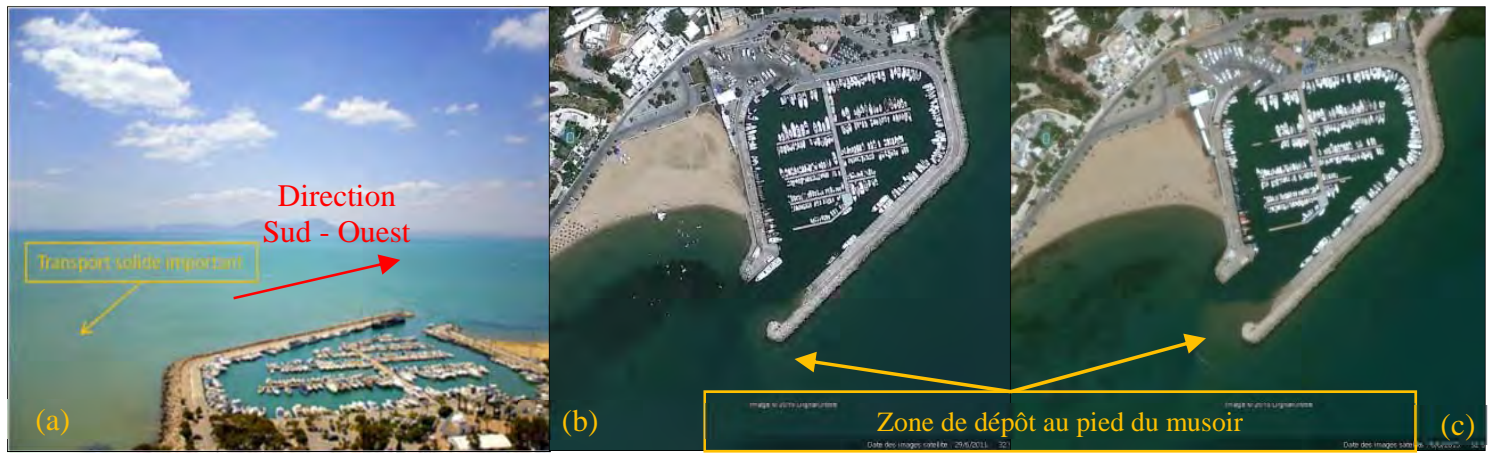

Figure 2. Vues aériennes avec marqueurs de transport sédimentaire :

(a) Transport solide important ; (b) état 2011 ; (c) état 2015.

\section{Modélisation}

Une série de calculs a été mise en oeœuvre pour étudier le fonctionnement hydrosédimentaire de la zone portuaire de Sidi Bou Saïd, analyser le phénomène d'ensablement de la passe d'entrée au port et vérifier l'impact des aménagements proposés. Cette chaine est constituée de trois principales étapes réalisées à l'aide des logiciels ARTEMIS, TELEMAC 2D et SISYPHE, qui modélisent respectivement l'agitation de la houle, les courants et le transport sédimentaire 
(http://docs.opentelemac.org/). Un domaine couvrant le Golfe de Tunis a été défini avec un maillage irrégulier particulièrement serré au niveau des zones d'intérêt. L'emprise du modèle représente environ $100 \mathrm{~km}$ de littoral et s'étend au large jusqu'à une trentaine de kilomètres des côtes. Les tailles des arêtes des éléments triangulaires qui constituent le maillage du modèle varient de $1000 \mathrm{~m}$ au large à moins de $100 \mathrm{~m}$ au niveau du littoral. Le maillage est fortement raffiné autour de Cap Carthage en général et autour du port en particulier avec des mailles de $20 \mathrm{~m}$. Le maillage du modèle est constitué de 31503 nœuds et de 61431 éléments (voir figure 3).

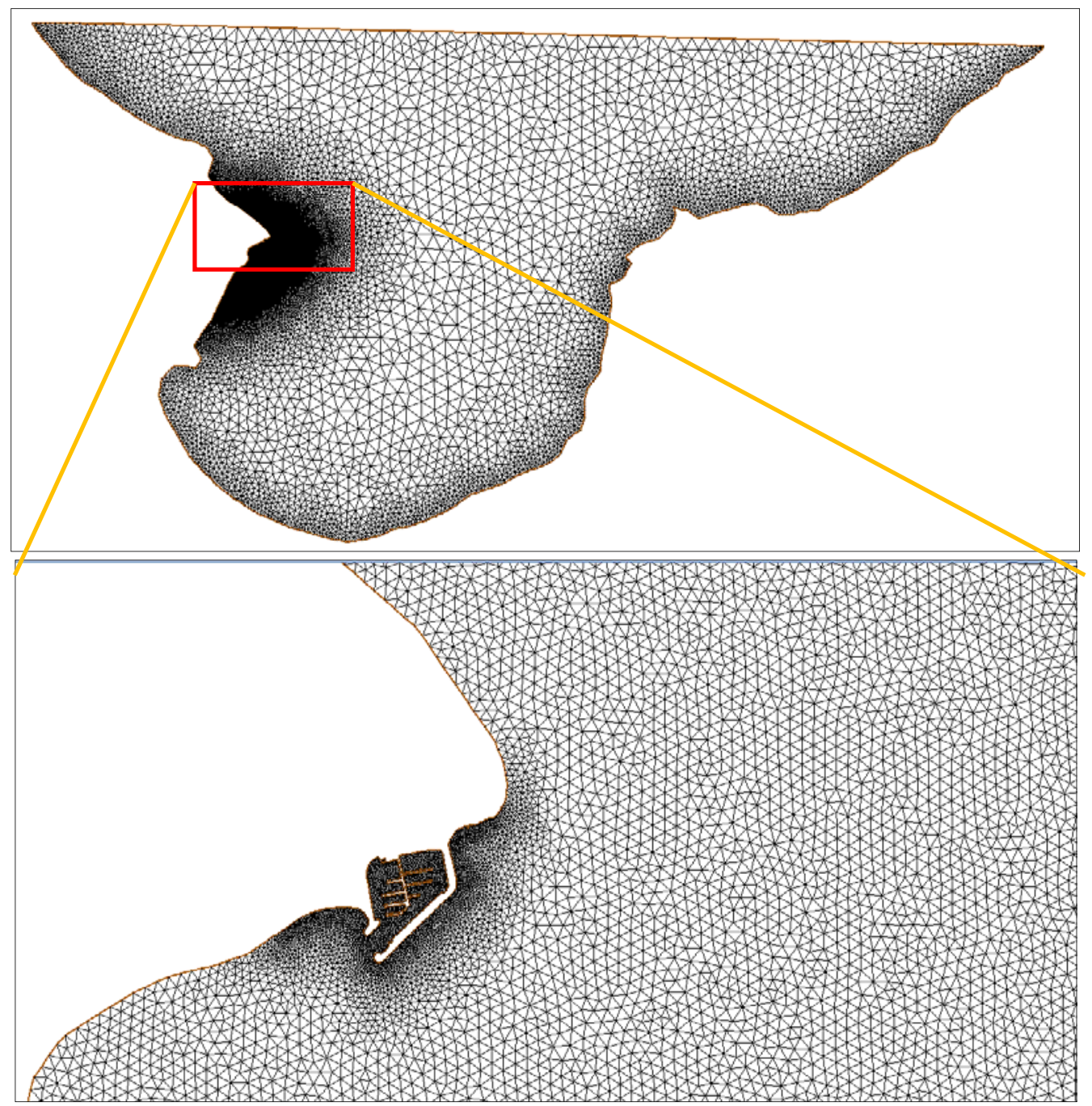

Figure 3. Maillage du modèle hydrodynamique et sédimentologique.

Les données de houles au large ont été définies à partir de la base de données UKMO (modèle météorologique global en Méditerranée) sur un point situé au large du golfe de Tunis (SAP, 2005). Les données de houles aux limites de la zone modélisée ont été établies à l'aide du modèle numérique de réfraction TOMAWAC. 
Côtes méditerranéennes menacées :

Risques et défis dans le contexte du changement climatique

\section{Résultats et aménagements proposés}

Le logiciel ARTEMIS appliqué à notre domaine d'étude a permis de montrer que pour les houles dominantes du secteur Nord, l'obliquité est très importante notamment au droit de la digue principale de l'ordre de $50^{\circ}$ (voir figure 4), ce qui correspond à une capacité de transit sédimentaire maximum. Les simulations des courants par le logiciel TELEMAC 2D, ont montré que le courant principal est relativement fort au droit de la digue principale avec une vitesse moyenne qui varie de $0,8 \mathrm{~m} / \mathrm{s}$ à $1 \mathrm{~m} / \mathrm{s}$ (voir : figure 5 et tableau 1). Ces courants, induits par la houle, transportent les sédiments vers le sud soit vers la plage attenante au port, ou encore en arrière du musoir obstruant ainsi la passe d'entrée.

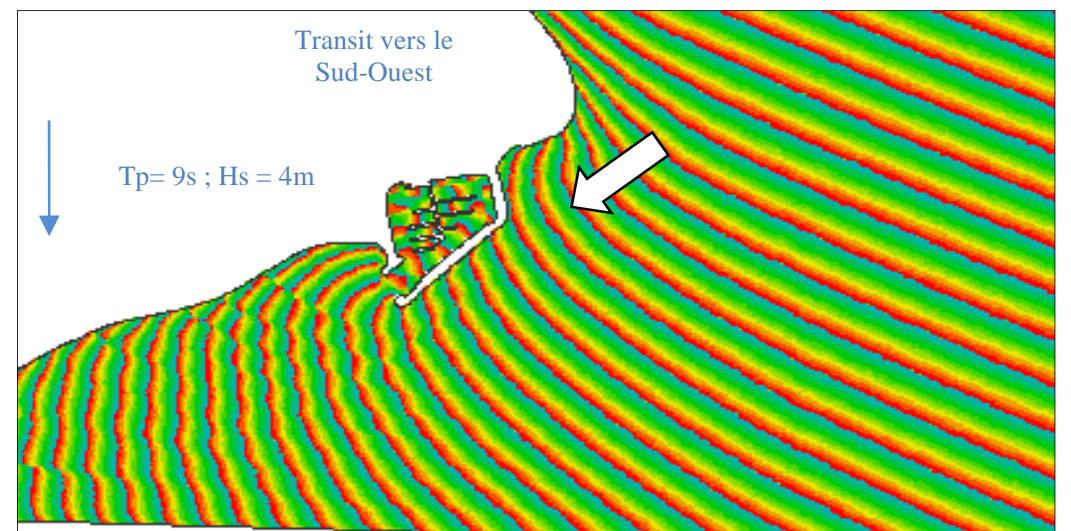

Figure 4. Phases de houle résultantes du modèle d'agitation pour une houle annuelle.

Ces conditions de courantologie relativement élevées couplées à l'obliquité de la houle induisent un transit sédimentaire vers le Sud-ouest estimé par les formules empiriques du CERC (CERC, 1984) et du LNH (J. Larras, R. Bonnefille, L. Perneker) entre $36345 \mathrm{~m}^{3} /$ an et $22172 \mathrm{~m}^{3} /$ an (voir tableau 2). Ces résultats sont cohérents avec les travaux réalisés dans la baie de Tunis (LOUATI \& ZARGOUNI, 2009).

Les aménagements proposés consistent à :

- Translater la digue principale et la contre digue de $120 \mathrm{~m}$ vers le Sud.

- Prolonger la digue principale de $50 \mathrm{~m}$.

Les résultats du modèle de courantologie montrent l'impact significatif des aménagements proposés sur les courants engendrés par l'action simultanée de la houle et de la marée. A l'encontre du comportement hydrosédimentaire de l'état actuel, les cartes des champs de vitesse pour l'état aménagé montrent un comportement assez différent en termes de vitesse et de direction des courants. En effet, les courants principaux sont redirigés vers le large. Un courant résiduel longe la première moitié de la digue principale vers le sud et le risque de dépôt se trouve au sud de la digue principale sur la façade extérieure. 


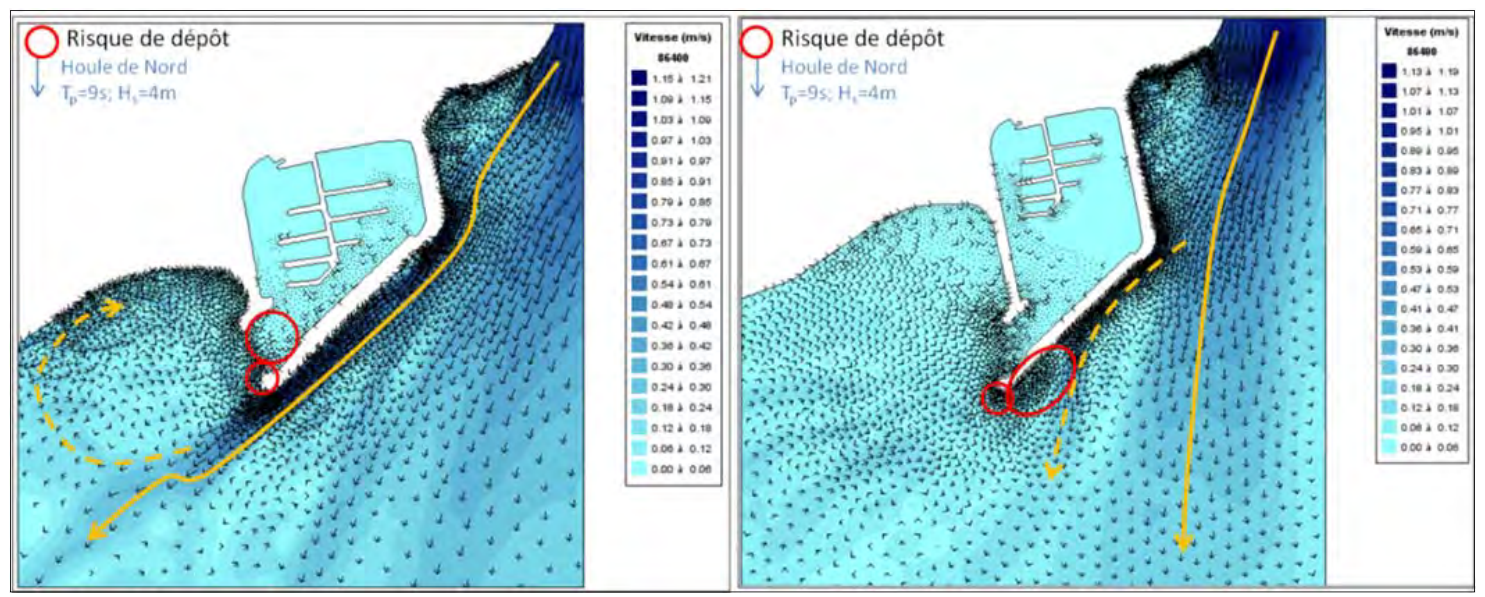

Figure 5. Courantologie associée à la houle de Nord : (a) état actuel ; (b) état aménagé.

Les vitesses du courant au droit de la digue principale passent de 0,9 m/s pour l'état actuel à $0,22 \mathrm{~m} / \mathrm{s}$ en état aménagé, soit une réduction de plus de $60 \%$ (voir tableau 1 ). Cette réduction s'explique par l'augmentation de la profondeur au droit de la digue principale qui passe de 2,0 m dans la situation actuelle à 4,5 m pour l'état aménagé.

Tableau 1. Vitesses des courants induits par la houle de direction Nord.

\begin{tabular}{lll}
\hline & Etat actuel & $\begin{array}{l}\text { Etat aménagé } \\
\boldsymbol{U}_{\text {moy }}(\boldsymbol{m} / \mathbf{s})\end{array}$ \\
\hline En amont du port & 0,38 & 0,40 \\
Au droit la digue principale & 0,90 & 0,22 \\
En aval du port & 0,22 & 0,04 \\
\hline
\end{tabular}

L’application du code SISYPHE a permis de déceler les tendances évolutives des fonds. Les résultats montrent qu'à l'état actuel, une zone de désordres avec une prédominance de l'érosion se présente à l'enracinement de la digue principale. Les sédiments arrachés dans ce secteur sont directement transportés vers l'extrémité du musoir et dans la passe du port qui constitue une zone de dépôts préférentielle.

Pour l'état aménagé on observe des dépôts dans l'axe Nord-Sud de la digue plus important que dans la situation actuelle, ce qui signifie que l'extension sur $120 \mathrm{~m}$ va permettre de créer un ensablement et une plage au Nord du port. Ce phénomène va interrompre le processus d'ensablement de la passe d'entrée et ce jusqu'à ce que la digue soit à nouveau contournée par le transit sédimentaire.

Des calculs de la capacité de transit sédimentaire ont été effectués en utilisant les formules empiriques du CERC et du LNH. Les résultats montrent que les aménagements proposés permettent de réduire cette capacité de plus de 50\% par rapport à l'état actuel (voir tableau 2). 
Côtes méditerranéennes menacées :

Risques et défis dans le contexte du changement climatique

Tableau 2. Capacité de transit sédimentaire calculée au droit de la digue principale.

\begin{tabular}{lllllll}
\hline & \multicolumn{2}{l}{ Etat actuel } & & \multicolumn{2}{c}{ Etat aménagé } \\
\cline { 2 - 3 } & CERC & LNH & & CERC & LNH \\
\hline Capacité de transport sédimentaire $\mathbf{Q}\left(\mathbf{m}^{3} / \mathbf{a n}\right)$ & 36345 & 22172 & & 16889 & 15619 \\
\hline
\end{tabular}

\section{Conclusion}

La modélisation numérique de l'état aménagé montre une nette modification de la situation en termes de courants et de transport sédimentaire. En effet le taux d'ensablement sera significatif au niveau de l'enracinement de la digue principale favorisant la création d'une plage de sable fin. On peut considérer que le transit sera interrompu à ce niveau sur une période de 5 à 10 ans ce qui constitue une garantie contre le risque d'ensablement de la passe d'entrée à moyen terme. S'agissant de l'ensablement de la passe d'entrée située dans des profondeurs plus importantes (4,5 m) elle ne sera pas affectée en termes de tirant d'eau avant une dizaine d'années minimum. Les dragages d'entretien seront réduits de moitié (50\%) par rapport aux dragages d'entretien actuels 5 à 10 ans après la réalisation des travaux. Donc si l'on considère une capacité de transport réduite de l'ordre de $2500 \mathrm{~m}^{3}$ /an, les opérations de dragage seront réalisées tous les 10 ans avec des quantités de $25000 \mathrm{~m}^{3}$. Le premier dragage sera effectué 15 à 20 années après la réalisation du projet.

\section{Références bibliographiques}

CERC -Coastal Engineering Research Centre- (1984). Shore Protection Manual, volumes I \& II. Department of the Army, US Army Corps of Engineers.

COMETE ENGINEERING (2008). Etude de protection et d'aménagement du versant Nord Est de la colline de Sidi Bou Saïd. Rapport pour l'Agence de Protection et d'Aménagement du Littoral de Tunisie.

LOUATI. M., ZARGOUNI F. (2009). Modélisation topo-bathymétrique et transit sédimentaire. Exemple des plages sableuses de la baie de Tunis, Nord-Est de la Tunisie. Géomorphologie : relief, processus, environnement, Vol. 3, pp 211-222. http://dx.doi.org/10.4000/geomorphologie.7686

SAP (2005). Marina de Gammarth "Étude d'agitation site 2A: Cap Gammarth à Salambo". Rapport d'étude inédit.

STUDI-SOGREAH (2003). Etude générale pour la protection du littoral tunisien contre l'érosion marine - Phase II : Hidrotecnica Portuguesa (1995). Rapport d'étude inédit. 